\title{
Effects of amoxicillin/clavulanic acid on the pharmacokinetics of valproic acid
}

This article was published in the following Dove Press journal:

Drug Design, Development and Therapy

10 August 2015

Number of times this article has been viewed

\author{
Soo-Yun Lee' \\ Wooseong $\mathrm{Huh}^{2}$ \\ Jin Ah Jung ${ }^{3}$ \\ Hye Min Yoo ${ }^{2}$ \\ Jae-Wook Ko ${ }^{1,2}$ \\ Jung-Ryul Kim²,4 \\ 'Department of Health Sciences and \\ Technology, SAIHST, Sungkyunkwan \\ University, ${ }^{2}$ Department of Clinical \\ Pharmacology and Therapeutics, \\ Samsung Medical Center, Seoul, \\ ${ }^{3}$ Department of Clinical Pharmacology, \\ Inje University, Busan Paik Hospital, \\ Busan, ${ }^{4}$ Department of Clinical \\ Research and Evaluation, SAIHST, \\ Sungkyunkwan University, Seoul, \\ Republic of Korea
}

\begin{abstract}
Valproic acid (VPA) is mainly metabolized via glucuronide, which is hydrolyzed by $\beta$-glucuronidase and undergoes enterohepatic circulation. Amoxicillin/clavulanic acid (AMC) administration leads to decreased levels of $\beta$-glucuronidase-producing bacteria, suggesting that these antibiotics could interrupt enterohepatic circulation and thereby alter the pharmacokinetics of VPA. This study aimed to evaluate the effects of AMC on the pharmacokinetics of VPA. This was an open-label, two-treatment, one-sequence study in 16 healthy volunteers. Two treatments were evaluated; treatment VPA, in which a single dose of VPA $500 \mathrm{mg}$ was administered, and treatment AMC + VPA, in which multiple doses of AMC 500/125 mg were administered three times daily for 7 days and then a single dose of VPA was administered. Blood samples were collected up to 48 hours. Pharmacokinetic parameters were calculated using noncompartmental methods. Fifteen subjects completed the study. Systemic exposures and peak concentrations of VPA were slightly lower with treatment AMC + VPA than with treatment VPA $\left(\mathrm{AUC}_{\text {last }}, 851.0 \mathrm{~h} \cdot \mathrm{mg} / \mathrm{L}\right.$ vs $889.6 \mathrm{~h} \cdot \mathrm{mg} / \mathrm{L} ; C_{\max }, 52.1 \mathrm{mg} / \mathrm{L}$ vs $\left.53.0 \mathrm{mg} / \mathrm{L}\right)$. There were no significant between-treatment effects on pharmacokinetics $(95 \%$ confidence interval [CI]) of $\mathrm{AUC}_{\text {last }}$ and $C_{\max }$ (95.7 [85.9-106.5] and 98.3 [91.6-105.6], respectively). Multiple doses of AMC had no significant effects on the pharmacokinetics of VPA; thus, no dose adjustment is necessary.
\end{abstract}

Keywords: drug-drug interaction, pharmacokinetics, enterohepatic circulation

\section{Introduction}

Valproic acid (VPA) is a widely used antiepileptic drug for the monotherapy or adjunctive therapy of simple or complex absence seizures. VPA is metabolized mainly by hepatic glucuronidation. ${ }^{1}$ In a process known as enterohepatic circulation (EHC), it is secreted via bile into the intestine, where it is hydrolyzed by enterobacteria to liberate the parent drug, which is then reabsorbed. Interestingly, amoxicillin/clavulanic acid (AMC) causes a marked decrease in the levels of anaerobic enterobacteria, especially bifidobacteria, lactobacilli, and clostridia, which could ultimately lead to altered pharmacokinetics for glucuronide drug conjugates. ${ }^{2}$ Indeed, a previous study reported significantly decreased concentrations of mycophenolic acid, which undergoes extensive EHC, following multiple administration of AMC. ${ }^{3}$

The goal of epilepsy treatment is to achieve a seizure-free status without adverse effects, which can be achieved by controlling VPA concentrations. Unfortunately, VPA has a narrow therapeutic window. ${ }^{4}$ During some seizures, people can injure themselves and develop other medical problems or life-threatening emergencies such as bruises, cuts, burns, and falls. ${ }^{5}$ So it is very important for them to maintain a constant concentration of VPA to prevent potentially life-threatening conditions arising from a failure to control drug concentrations. For this reason, VPA concentration is 
monitored to adjust dosage regimens and to achieve the desired clinical effects while avoiding toxicity.

To date, there are no published drug-drug interaction studies of VPA and AMC, although they are commonly used as first-line treatments for each therapeutic indication. This study aimed to evaluate the effects of AMC on the pharmacokinetics of VPA following multiple oral administration of $\mathrm{AMC}$ in healthy subjects.

\section{Methods \\ Study design}

This was an open-label, two-treatment, single-sequence, drug interaction study. This study was coordinated by the Clinical Trial Center of Samsung Medical Center (Seoul, Republic of Korea). Healthy male subjects between the ages of 19 years and 55 years with a body mass index (BMI) of $19-27 \mathrm{~kg} / \mathrm{m}^{2}$ (extremes included) were eligible for enrollment.

On day 1 , subjects received a single dose of VPA $500 \mathrm{mg}$ (Depakote ${ }^{\circledR}$; Abbott Laboratories) with $240 \mathrm{~mL}$ of water. After washout of 5-9 days, subjects received AMC 500/125 mg (Augmentin ${ }^{\circledR}$; GlaxoSmithKline plc) three times daily for 7 days. On day 15, subjects received a single dose of VPA and were then confined to the center from the morning of day 16. The highest approved dosage of each drug was used in this study to maximize the ability to detect a significant pharmacokinetic interaction. This AMC dose was considered sufficient for affecting the intestinal microbiota based on a previous study. ${ }^{6}$

The study was approved by the Institutional Review Board of Samsung Medical Center. The study was conducted in accordance with Good Clinical Practice and the Declaration of Helsinki. The study is registered at cris.nih.go.kr/cris (KCT0001165). Written informed consent was obtained from all participants prior to screening evaluations.

\section{Blood sampling}

Venous blood samples were collected at 0 hour (predose), 2 hours, 2.67 hours, 3.33 hours, 4 hours, 5 hours, 6 hours, 7 hours, 9 hours, 12 hours, 24 hours, and 48 hours after study drug administration (in two subjects, samples were obtained at 1 hour and 3 hours instead of 2.67 hours and 3.33 hours, respectively). Samples, placed in heparinized tubes, were centrifuged at $2,000 \times g$ for 10 minutes at $4^{\circ} \mathrm{C}$. Plasma samples were stored at $-70^{\circ} \mathrm{C}$ until further bioanalysis.

\section{Drug analysis}

Assays for plasma VPA concentrations were performed with a validated fluorescence polarization immunoassay (COBAS INTEGRA ${ }^{\circledR} 800$; Roche Diagnostics, Mannheim,
Germany) by duly licensed laboratories. Assays were conducted in compliance with applicable standard operating procedures in place at the time of analysis in the Clinical Chemistry Laboratory of Samsung Medical Center. The assay had a coefficient of variation lower than $2.4 \%$ and a sensitivity of $3.15 \mathrm{mg} / \mathrm{L}$. The lower limit of quantification was $1.59 \mathrm{mg} / \mathrm{L}$, and calibration ranged from $1.59 \mathrm{mg} / \mathrm{L}$ to $35 \mathrm{mg} / \mathrm{L}$.

\section{Safety analysis}

Safety evaluations included observations of any clinically significant changes in physical examinations, clinical laboratory tests (blood chemistry, hematology and urinalysis), and vital signs (systolic/diastolic blood pressure and pulse rate). Blood pressure values were obtained in the seated position with the upper arm using a semi-automated cuff-oscillometric sphygmomanometer. Adverse events (AEs) were monitored; evaluated for the frequency, severity, and relationship to treatment; and recorded using $\operatorname{MedDRA}^{\circledR}$ (Version 16.0; Maintenance and Support Services Organization, Chantilly, VA, USA).

\section{Pharmacokinetic analysis}

The pharmacokinetic parameters of VPA were calculated by noncompartmental analysis of individual plasma concentration-time data using Phoenix ${ }^{\circledR}$ WinNonlin ${ }^{\circledR}$ 6.3 software (Certara, St Louis, MO, USA). The maximum concentration $\left(C_{\max }\right)$ and the time to $C_{\max }\left(t_{\max }\right)$ were obtained from the observed data. The area under the plasma concentration-time curve from time 0 to the last quantifiable concentration $\left(\mathrm{AUC}_{\text {last }}\right)$ and the partial AUC from 4 hours to 12 hours $\left(\mathrm{AUC}_{4-12}\right)$ as an estimate of EHC were determined using the linear/log trapezoidal method. The elimination half-life $\left(t_{1 / 2}\right)$ was calculated as: $t_{1 / 2}=\ln (2) / k_{\mathrm{el}}$, where $k_{\mathrm{el}}$ was the elimination rate constant. $k_{\mathrm{el}}$ was calculated by linear regression of the log-linear concentration-time curve.

\section{Statistical methods}

All statistical analyses were conducted using $\mathrm{SAS}^{\circledR}$ Enterprise Guide $^{\circledR} 4.3$ software (SAS Institute Inc., Cary, NC, USA). The $C_{\max }$ and $\mathrm{AUC}_{\text {last }}$ estimates were log-transformed and then analyzed with a generalized linear model. Back-transformed summary statistics includes geometric least-squares means and the geometric mean ratio (GMR) for betweentreatment comparisons with $90 \%$ confidence intervals (CIs). The analysis of $t_{\max }$ was based on untransformed data using non-parametric methods (Wilcoxon-signed rank test).

A sample size of 16 was calculated to be sufficient (with a power of $90 \%$ at the $5 \%$ significance level), assuming 
Table I Treatment-emergent adverse events

\begin{tabular}{llllll}
\hline & VPA alone $(\mathbf{n}=\mathbf{I 6})$ & & & AMC + VPA $(\mathbf{n}=\mathbf{I 6})$ \\
\cline { 2 - 3 } \cline { 5 - 6 } & Number of events & Number $(\%)$ of subjects & Number of events & Number $(\%)$ of subjects \\
\hline Diarrhea & $\mathrm{I}$ & $\mathrm{I}(6.25)$ & 3 & $3(18.75)$ \\
Dyspepsia & 0 & $0(0.00)$ & $\mathrm{I}$ & $\mathrm{I}(6.25)$ \\
Asthenia & 0 & $0(0.00)$ & $\mathrm{I}$ & $\mathrm{I}(6.25)$ \\
Increased blood bilirubin & 0 & $0(0.00)$ & $\mathrm{I}$ & $\mathrm{I}(6.25)$ \\
Increased blood creatine phosphokinase & 0 & $0(0.00)$ & 2 & $2(12.50)$ \\
Decreased neutrophil count & $\mathrm{I}$ & $\mathrm{I}(6.25)$ & $\mathrm{I}$ & $\mathrm{I}(6.25)$ \\
Headache & 0 & $0(0.00)$ & $\mathrm{I}$ & $\mathrm{I}(6.25)$ \\
Hypoesthesia & 0 & $0(0.00)$ & $\mathrm{I}$ & $\mathrm{I}(6.25)$ \\
Presyncope & 0 & $0(0.00)$ & $\mathrm{I}$ & $\mathrm{I}(6.25)$ \\
Somnolence & 0 & $0(0.00)$ & $\mathrm{I}$ & $\mathrm{I}(6.25)$ \\
Nocturia & 0 & $0(0.00)$ & $\mathrm{I}$ & $\mathrm{I}(6.25)$ \\
Total & 2 & $2(12.50)$ & $\mathrm{I}$ & $9(56.25)$
\end{tabular}

Abbreviations: VPA, valproic acid; AMC, amoxicillin/clavulanic acid.

a difference of $20 \%$ in exposure to VPA with or without $\mathrm{AMC}$ and an intrasubject coefficient of variation of $15 \%$ for VPA.

\section{Results}

Twenty-five volunteers were screened, and 17 of them were randomized. Two subjects prematurely discontinued the study following withdrawal of informed consent. A total of 15 subjects completed the study. Subjects $(n=17)$ had a mean age of 28.5 years (range, 21.0-45.0 years), a mean weight of $70.2 \mathrm{~kg}$ (range, $58.6-79.8 \mathrm{~kg}$ ), and a mean BMI of $23.4 \mathrm{~kg} / \mathrm{m}^{2}$ (range, $\left.19.4-27.0 \mathrm{~kg} / \mathrm{m}^{2}\right)$. They had no significant medical history and did not take any medication. Mean systolic and diastolic blood pressures were $124.1 \mathrm{mmHg}$ (range, $110.0-144.0 \mathrm{mmHg}$ ) and $76.1 \mathrm{mmHg}$ (range, $68.0-91.0 \mathrm{mmHg}$ ), respectively. The mean pulse rate was $69.8 \mathrm{bpm}$ (range, 49.0-93.0 bpm).

\section{Safety}

Treatment-emergent AEs are shown in Table 1. Nine subjects (56.3\%) reported 14 AEs when coadministered with AMC and two subjects (12.5\%) reported two AEs when administered VPA alone. Six subjects (37.5\%) had seven events that were considered drug related: diarrhea (4), dyspepsia (1), decreased neutrophil count (1), and nocturia (1). All AEs were considered to be mild in severity and resolved without sequelae. The most frequently reported $\mathrm{AE}$ was diarrhea. Three subjects had clinically significant laboratory abnormalities: increased blood bilirubin, increased blood creatine phosphokinase, and decreased neutrophil count. There were no clinically significant changes in vital signs.

\section{Pharmacokinetics}

Mean plasma VPA concentration-time profiles are shown in Figure 1. After reaching $C_{\max }$, plasma VPA concentrations appeared to decline in a monophasic manner. Pharmacokinetic parameters and statistical comparisons of VPA are shown in Table 2. When coadministered with $\mathrm{AMC}$, the $C_{\max }$ and $\mathrm{AUC}_{\text {last }}$ of VPA decreased by $1.5 \%$ and $4.4 \%$, respectively. There was no difference in the partial $\mathrm{AUC}_{4-12}$ between treatments. The median $t_{\max }$ value was comparable between treatments. The mean $t_{1 / 2}$ value was approximately 16 hours in both treatments. The GMRs with $90 \%$ CIs for the $\mathrm{AUC}_{\text {last }}, \mathrm{AUC}_{4-12}$, and $C_{\max }$ fell entirely within a range of $0.80-1.25$, which indicates no significant interaction with $\mathrm{AMC}$.

\section{Discussion and conclusion}

In this study, visual observations revealed that plasma concentrations of VPA showed only one peak at approximately

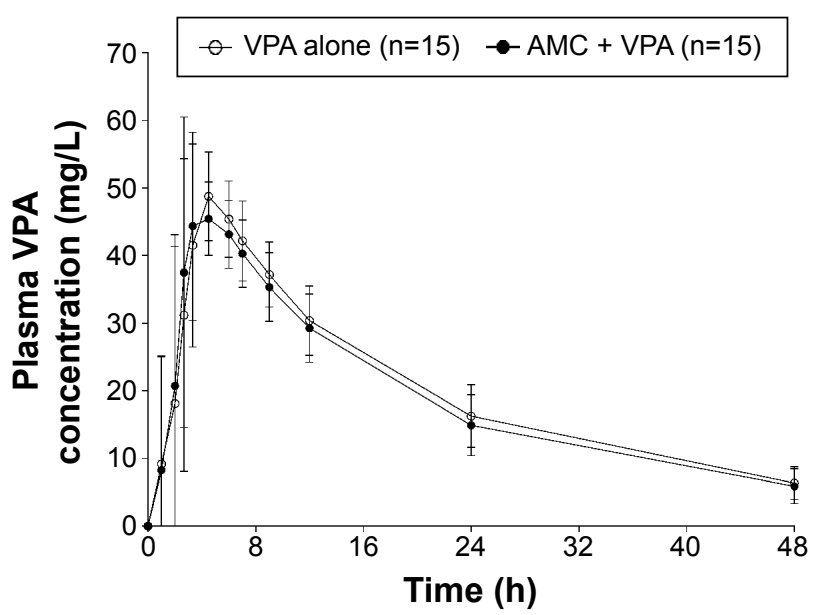

Figure I Mean plasma VPA concentration-time profiles with and without AMC in healthy subjects.

Note: Error bars represent standard deviation.

Abbreviations: h, hour; VPA, valproic acid; AMC, amoxicillin/clavulanic acid. 
Table 2 Pharmacokinetic parameters and statistical comparisons of VPA with and without AMC in healthy subjects

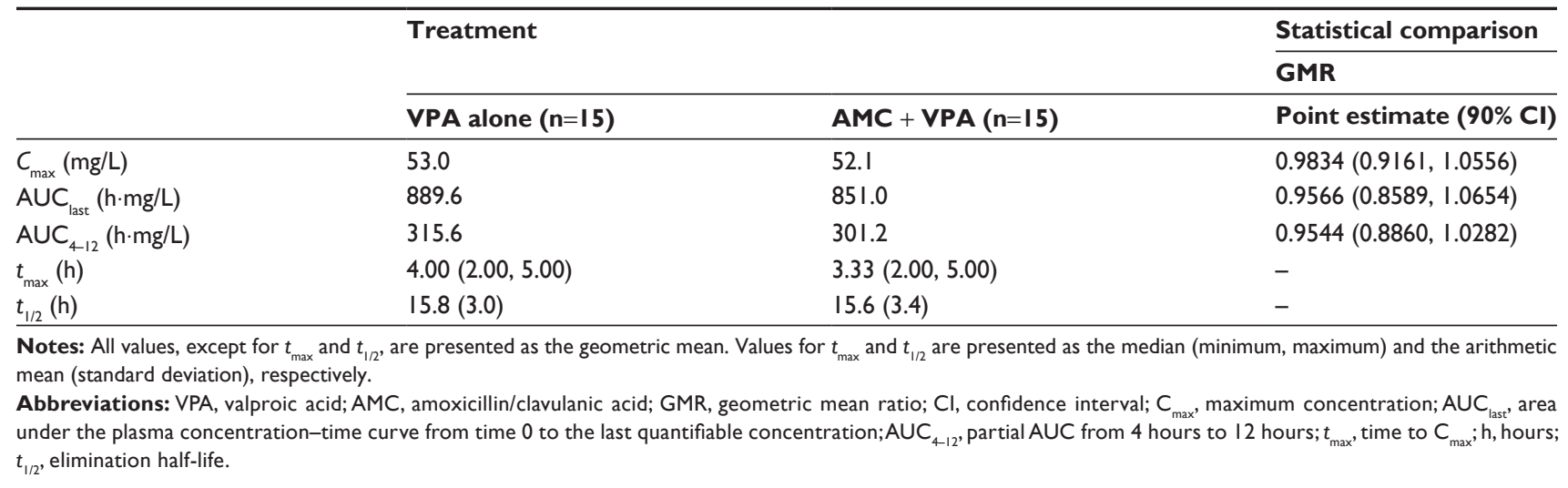

2-5 hours, regardless of the administration of AMC. This result is not consistent with a previous study in which mean VPA profiles for both sexes showed a marked secondary peak at 8 hours, implying the presence of a secondary absorption process. ${ }^{7}$ Note that EHC may not be the only determinant factor for the appearance of multiple peaks in plasma concentration. Indeed, multiple peaks in the concentration-time curve can occur as a consequence of a number of different mechanisms. ${ }^{8}$ For VPA, there are two possible causes for the presence of a secondary peak: formulation factors and physiological factors (EHC). ${ }^{8}$ First, because we selected the same delayed-release formulation as the previous study, the presence of a secondary peak cannot be explained by the formulation. ${ }^{7}$ For sustained-release formulations of VPA, the secondary peak can result from alterations in release and dissolution rates at different gastrointestinal sites and from alterations in absorption rates. ${ }^{8}$ Thus, delayed-release tablets are suitable for modeling EHC of VPA, as mentioned in the previous study. ${ }^{7}$ Second, various species exhibit differences in EHC of VPA. It is well known that, in rats, VPA displays secondary peaks, suggesting the presence of EHC in the disposition of VPA. ${ }^{9,10}$ In monkeys, the excretion into the bile was very limited, although the amount of conjugated VPA was comparable to that in rats. ${ }^{9}$ Thus, considering the close genetic relatedness between humans and monkeys, VPA may not undergo extensive EHC in humans, although the major metabolites of VPA are the same glucuronide conjugates observed in rats.

According to the previous study, women had a larger AUC and a higher $C_{\text {max }}$ of VPA than men; however, when correction for body weight was made, such differences disappeared. ${ }^{7}$ It was also observed for mycophenolic acid, with a marked difference in the ratio of mycophenolic acid to its glucuronide conjugates between sexes. ${ }^{11}$ In men, due to the reduced expression of efflux pumps, saturation of hepatobiliary transporters would result in longer drug residence in hepatocytes and thus faster clearance, ultimately decreasing the reabsorbed fraction. ${ }^{7}$ Furthermore, because VPA glucuronide conjugates are found in urine, further studies are needed to clarify the role of EHC in metabolism of VPA, considering the potential sex differences in its metabolism, measured directly by using urine samples. ${ }^{12}$

We did not evaluate changes in the number or type of enterobacteria using fecal samples. On the basis of previous studies, however, multiple doses of amoxicillin for 7 days were sufficient to reduce enterobacteria levels. ${ }^{2,6}$

The incidence of AEs when coadministered VPA with AMC was reported higher compared with that of administration of VPA alone, even considering multiple AMC administration.

In summary, multiple AMC administration had little effect on the pharmacokinetics of VPA, indicating that dose adjustments of VPA may not be required under these circumstances. Further studies are necessary to determine the clinical impact on patients treated with VPA.

\section{Acknowledgment}

This study was supported by a Samsung Medical Center grant (SMO1132061).

\section{Disclosure}

The authors report no conflicts of interest in this work.

\section{References}

1. Dickinson RG, Hooper WD, Eadie MJ. pH-dependent rearrangement of the biosynthetic ester glucuronide of valproic acid to betaglucuronidase-resistant forms. Drug Metab Dispos. 1984;12(2):247-252.

2. Lode H, Von der Hoh N, Ziege S, Borner K, Nord CE. Ecological effects of linezolid versus amoxicillin/clavulanic acid on the normal intestinal microflora. Scand J Infect Dis. 2001;33(12):899-903. 
3. Borrows R, Chusney G, Loucaidou M, et al. The magnitude and time course of changes in mycophenolic acid 12-hour predose levels during antibiotic therapy in mycophenolate mofetil-based renal transplantation. Ther Drug Monit. 2007;29(1):122-126.

4. Hiemke C, Baumann P, Bergemann N, et al. AGNP consensus guidelines for therapeutic drug monitoring in psychiatry: update 2011. Pharmacopsychiatry. 2011;44(6):195-235.

5. Epilepsy.org.uk [homepage on the Internet]. Epilepsy Action; c2015 [cited Jun 24, 2015]. Available from: http://www.epilepsy.org.uk/. Accessed Jun 26, 2015.

6. Koning CJ, Jonkers DM, Stobberingh EE, Mulder L, Rombouts FM, Stockbrugger RW. The effect of a multispecies probiotic on the intestinal microbiota and bowel movements in healthy volunteers taking the antibiotic amoxycillin. Am J Gastroenterol. 2008;103(1):178-189.

7. Ibarra M, Vazquez M, Fagiolino P, Derendorf H. Sex related differences on valproic acid pharmacokinetics after oral single dose. J Pharmacokinet Pharmacodyn. 2013;40(4):479-486.
8. Davies NM, Takemoto JK, Brocks DR, Yanez JA. Multiple peaking phenomena in pharmacokinetic disposition. Clin Pharmacokinet. 2010;49(6): 351-377.

9. Dickinson RG, Harland RC, Ilias AM, et al. Disposition of valproic acid in the rat: dose-dependent metabolism, distribution, enterohepatic recirculation and choleretic effect. J Pharmacol Exp Ther. 1979;211(3): 583-595.

10. Singh K, Orr JM, Abbott FS. Pharmacokinetics and enterohepatic circulation of 2-n-propyl-4-pentenoic acid in the rat. Drug Metab Dispos. 1988; 16(6):848-852.

11. Morissette P, Albert C, Busque S, St-Louis G, Vinet B. In vivo higher glucuronidation of mycophenolic acid in male than in female recipients of a cadaveric kidney allograft and under immunosuppressive therapy with mycophenolate mofetil. Ther Drug Monit. 2001;23(5):520-525.

12. Kuhara T, Hirokata Y, Yamada S, Matsumoto I. Metabolism of sodium dipropylacetate in human. Eur J Drug Metab Pharmacokinet. 1978; 3(3):171-177.

\section{Publish your work in this journal}

Drug Design, Development and Therapy is an international, peerreviewed open-access journal that spans the spectrum of drug design and development through to clinical applications. Clinical outcomes, patient safety, and programs for the development and effective, safe, and sustained use of medicines are a feature of the journal, which has also been accepted for indexing on PubMed Central. The manuscript management system is completely online and includes a very quick and fair peer-review system, which is all easy to use. Visit http://www.dovepress.com/testimonials.php to read real quotes from published authors.

Submit your manuscript here: http://www.dovepress.com/drug-design-development-and-therapy-journal 